\title{
Calidad de vida percibida y su relación con la práctica de actividad física en el ámbitolaboral. Un estudiopiloto \\ Perceived quality of life and its correlation to the practice of physical activity in the work sphere. Pilot study

\author{
Joaquín Martínez San Estebany África Calvo Lluch \\ Universidad Pablo de Olavide
}

\begin{abstract}
Resumen: El objetivo del presente estudio trata de identificar la relación existente entre la calidad de vida percibida relacionada con la salud, y la práctica de actividad física realizada de manera habitual en un grupo de empleados públicos. Método. Un total de 125 trabajadores del Servicio de salud del Ayuntamiento de Sevilla cumplimentaron el cuestionario de salud SF-36 versión 2 AGUDA para determinar su calidad de vida relacionada con la salud en el ámbito laboral, así como un cuestionario de preguntas de carácter general indicando el tipo y frecuencia de actividad física que realizaban. Resultados. Para todas las dimensiones del cuestionario, el estado de salud está dentro del rango promedio, siendo las dimensiones de función física y vitalidad las que presentan una mejor puntuación. Conclusión. Los trabajadores que no realizan actividad física tienen una peor percepción de su salud o por lo menos comparada con la del año anterior, mientras que los que si realizan actividad física de manera regular presentan respuestas indicadoras de una percepción más positiva de su calidad de vida relacionada con su salud.
\end{abstract}

Palabras clave: Calidad de vida relacionada con la salud (CVRS), calidad de vida laboral (CVL), actividad física, SF-36 versión 2 AGUDA, escalas o dimensiones de la salud.

Abstract: The aim of this study is to identify the correlation between perceived quality of life regarding health and the regular practice of physical exercise, in a group of public sector workers. Method: A total of 125 workers from the Seville Council Health Service completed the SF-36 version 2 AGUDA health survey, in order to rate their quality of life regarding health in the work sphere, along with a general question survey indicating the type and frequency of the physical activity they do. Results: In all parts of the survey the state of health falls within the average range, the highest scores being those of physical function and vitality. Conclusion: The workers who do no physical exercise have a worse perception of their health, at least compared with that of last year, while those who do regular physical activity score more positive results concerning their perception of their quality of life in terms of health.

Keywords: Quality of life regarding health (QLRH), quality of working life, physical activity, SF-36 version 2 AGUDA, scales or dimensions of health.

\section{Introducción}

\section{Calidad de vida}

El concepto de calidad de vida(CV) ha comenzado a utilizarse cada vez más en el campo de las evaluaciones en salud o como medida de bienestar. Pese a esto, no existe una definición única del concepto ni una completa diferenciación con otros términos similares, siendo frecuentemente mal utilizada. (Urzúa \& Caqueo-Urízar, 2012).

Según la OMS, la CV se define como la percepción del individuo sobre su posición en la vida dentro del contexto cultural y el sistema de valores en el que vive y con respecto a sus metas, expectativas, normas y preocupaciones. Es un concepto extenso y complejo que engloba la salud física, el estado psicológico, el nivel de independencia, las relaciones sociales, las creencias personales y la relación con las características sobresalientes del entorno. Esta definición pone de manifiesto criterios que afirman que la (CV) se refiere a una evaluación subjetiva, con dimensiones tanto positivas como negativas, y que está arraigada en un contexto cultural, social y ambiental. (WHOQOL- OMS, 1996).

La(CV) en el ámbito laboral es un tema de actualidad en la gestión del factor humano de las organizaciones modernas. Básicamente se refiere al carácter positivo o negativo de un ambiente laboral así como a las ventajas o desventajas que representan para las personas que lo integran. El termino (CVL) o Calidad de vida en el trabajo que traducido al inglés quiere decir Quality of Work Life (QWL), tuvo sus orígenes en una serie de conferencias patrocinadas al final de los años 60 y comienzos de los 70 por el Ministerio de Trabajo de los EE.UU. y la Fundación FORD. Estas conferencias fueron estimuladas por el fenómeno de la alienación del trabajador simbolizado por las huelgas entre la población activa mayoritariamente joven de una nueva planta de montaje de la General Motors, en Ohio. (Gibson, Ivanicevich \& Donelly, 1996).

Fecha recepción: 22-03-13- Fecha envío revisores: 06-06-13- Fecha de aceptación: 09-08-13 Joaquín Martínez San Esteban

Calle El Balcón, n ${ }^{\circ} 70$ - Urbanización Sport Aljarafe

41940 Tomares (Sevilla)

sujuaqui@gmail.com
La (CVL) es un sentimiento de satisfacción y bienestar que la persona trabajadora experimenta como consecuencia de su ajuste a realidades correspondientes al trabajo que desempeñan y a su contexto, o, como el grado en que la actividad laboral que una persona realiza está organizada para contribuir a sumás completo desarrollo como ser humano. Hay que tener en cuenta que ese grado es resultado de una evaluación que, enúltimo extremo, debe hacer la propia persona (Rodríguez-Marín, 2010).

Un estilo de vida saludable se concibe como un recurso valioso para reducir la incidencia y el impacto de los problemas de salud, para la recuperación de los mismos, para hacer frente a factores estresantes en la vida y para mejorar la (CV), constituyendo una adaptación del individuo a su entorno social. El ejercicio y la actividad física de intensidad moderada y realizados regularmente resultan un factor esencial en un estilo de vida saludable, orientado, junto a otros factores de riesgo, especialmente a minimizar los efectos del denominado síndrome metabólico, contribuyendo al apoyo de un desarrollo social positivo (interacción eintegración), a la prevención de enfermedades (osteoporosis, hipertensión, obesidad, ansiedad y depresión) y a la mejora de la salud pública, reduciendo con ello gastos sanitarios y aumentando la productividad de la población en general. (León Prados et al., 2011). En este sentido, teniendo en cuenta la edad de jubilación actual en el ámbito laboral y su probable aumento, la práctica de actividad física en el colectivo de personas mayores a partir de los 60 años, debe convertirse en una obligación, estando ello respaldado no solo por numerosos estudios, sino también por administraciones, las cuales consideran de gran importancia la presencia de programas de actividad física dirigidos a este sector de la población. (Martínez, Cocca, Mohamed \& Ramírez, 2010).

Instrumentos de medida de la salud y calidad de vida relacionada con la salud (CVRS)

En los últimos años ha aumentado el interés en la medida de la salud y de la CVRS, tanto en investigación como en la práctica clínica. Los usuarios de instrumentos de medida sobre la CVRS consideran la información que aportan como útil e instructiva. (Alonso, Prieto \& Antó, 1995). 
Los instrumentos de medida del estado de salud pueden clasificarse en dos grandes tipos: genéricos y específicos. Mientras que los específicos están diseñados para su utilización en pacientes con un tipo de problema de salud concreto (p.ej., asma), los genéricos son independientes del diagnóstico y se han desarrollado para poder ser utilizados en diferentes tipos de pacientes o poblaciones, ya sea el caso deuna población laboral. Entre las ventajas de los instrumentos genéricos destaca la posibilidad de comparar el impacto de las diferentes dimensiones de estudio (Vilagut, et al., 2008), así como la de obtener valores poblacionales de referencia. (Alonso, et al., 1995).

De los diferentes instrumentos de medida existentes podríamos destacar como específicos el PECVEC, CAVIDRA, AGHDA, MOSHIV, IPSS o el VF-14 y como genéricos el GHQ-28 y su versión breve GQH-12, NHP, SIP, APGAR, EuroQol-5D, SF-6D, SF-8, SF-12 o el SF-36 y su versión 2, esteúltimo siendo el mas utilizado en España para este tipo de estudios. (Badia \& Baró, 2001).

\section{La versión 2 del cuestionario SF-36 (SF-36 v2)}

Como ampliación a la versión 1 del cuestionario SF-36, en 1996 se desarrolló la versión 2.0 del cuestionario original SF-36, con el principal objetivo de mejorar las características métricas de las 2 escalas, Rol emocional y Rol físico, a través de la clasificación de los 36 ítems del cuestionario en las 8 dimensiones (Función física, Rol físico, Dolor corporal, Salud general, Vitalidad, Función social, Rol emocional y Salud mental), así como en las 2 componentes sumario (Física y Mental).

Para cada una de las 8 dimensiones, los ítems son codificados, agregados y transformados en una escala que tiene un recorrido desde 0 (el peor estado de salud para esa dimensión) hasta 100 (el mejor estado de salud).Las puntuaciones se calibran demodo queel 50 es lapuntuación media o normal. Este resultado permite la comparación entre tres encuestas y en los más de 17.000 estudios publicados en los últimos 20 años. Esta bibliografía incluye el estudio de cientos de enfermedades, condiciones y poblaciones, y mejora notablemente la capacidad de interpretar los datos de la encuesta del cuestionario de salud SF-36 para nuevos estudios.

\section{Propósito de la investigación}

Objetivos del presente estudio:

1- Conocer los aspectos más importantes de la (CVRS) en trabajadores pertenecientes a una administración pública promotora de la salud.

2- Determinar qué trabajadores tienen una mejor percepción de su CVRS, teniendo en cuenta las variables, sexo, grupo de edad y nivel de estudios.

3-Conocer qué tipo de actividad física realizan los trabajadores de dicho servicio de Salud del Ayuntamiento de Sevilla.

\section{Revisión bibliográfica (SF-36)}

Se realiza una revisión bibliográfica nacional e internacional de artículos publicados en los últimos 5 años en diferentes bases de datos como Physical Education Journals (ProQuest), ProQuest Health and Medical Complete, SPORTdiscus y Google Académico, la búsqueda se completó con una segunda revisión específica para el ámbito laboral. El volumen total de artículos encontrados fue de 323 de los cuáles 34 han sido utilizados para realizar el presente trabajo, el resto fue excluido al no cumplir el requisito de artículo científico (publicaciones, Posters, Seminarios o Simposiums), no utilizar el cuestionario SF-36 o hacer reseñas históricas del mismo, faltas de rigor o repetidas en otros artículos.

\section{Material y método \\ Diseño}

Se trata de un estudio no experimental de tipo transversal, porque con el instrumento diseñado y validado, identificamos y observamos una situación existente y no provocada en un solo momento y en un tiempo único, observando los fenómenos a estudiar en su ambiente natural para después analizarlos, no existiendo ni manipulación intencional ni asignación al azar, pues los sujetos estudiados ya pertenecían a un grupo o nivel determinado, es decir, describimos la (CV) de un grupo de trabajadores del Ayuntamiento de Sevilla en su lugar de trabajo.

Lametodología de investigación es detipo descriptiva y correlacional, ya que el estudio no se limita a describir la población en función de las variables seleccionadas en el cuestionario entregado, sino que, además, explora algunas correlaciones relacionadas entre los componentes de la (CV), el tipo de actividad física más realizado, el sexo, la edad o el nivel de estudios, midiendo o evaluando diversos aspectos, dimensiones o componentes del fenómeno a estudiar, en este caso, la (CVRS) en el ámbito laboral.

\section{Muestra}

Un total de 183 trabajadores componen la Relación de Puestos de Trabajo (RPT) en el año 2011 del Servicio de Salud, dependiente de la Dirección General de Familia y Salud dentro del Área deFamilia,Asuntos Sociales y Zonas de Especial Actuación del Excmo. Ayuntamiento de Sevilla. Del total de trabajadores de la RPT han participado finalmente en el estudio 125 (n).

Se ha determinado una muerte muestral de 58 sujetos, motivada principalmente al encontrarse ausentes de su puesto de trabajo durante el periodo de entrega de los cuestionarios, principalmente por baja laboral, e incluyendo a 5 sujetos que no quisieron realizar dicho cuestionario por motivos personales.

\section{Instrumentos}

Los instrumentos utilizados en este trabajo para la realización del estudio como para el posterior análisis de los mismos fueron los siguientes: Cuestionario de Salud SF-36 Versión 2 AGUDA. Versión Española de SF-36 v2 TM Health Survey - 1996, 2000 adaptada por J. Alonso y Cols., 2003, cuestionario de preguntas de carácter general indicando edad, sexo, nivel de estudios y actividad física realizada de manera regular (2-3 veces por semana). Para el análisis de los datos se ha utilizado el programa estadístico SPSS versión 18.0 para Windows y una aplicacióninformática con algoritmos decálculo para las puntuaciones de las 8 dimensiones y componente sumario http:// www.qualitymetric.com/demos/TP_Launch.aspx?SID=100 (acceder a demo).

\section{Procedimiento}

Se hace entrega del cuestionario en papel y se recoge por el propio autor, verificándose su correcta cumplimentación.

La entrega de los mismos se realizó durante la $4^{\mathrm{a}}$ semana de enero y $1^{\text {a }}$ de febrero de 2012 recogiéndose el último cuestionario el 29 de febrero de 2012. Junto con el cuestionario se entregó una cartaautorización donde se informaba sobre la estricta confidencialidad y el carácter anónimo de los datos y resultados recogidos en el cuestionario, objetivo y finalidad del estudio, autorizando la entrega por parte del encuestador para la posterior cumplimentación por todos los trabajadores de este Servicio.

\section{Resultados}

De los resultados del cuestionario de preguntas de carácter general cabe destacar que de los trabajadores del Servicio de Salud del Ayuntamiento de Sevilla, un 52.8\% son mujeres, un 81.3\% tiene entre 41-65 años, un 63.1\% tiene estudios universitarios y para un 58\% la actividad física más realizada es caminar rápidamente, correr, patinar, senderismo o montar en bicicleta.

Los resultados del cuestionario SF-36 v2 muestran que un 80.8\% de los trabajadores consideran que en general su salud es excelente, buena o muy buena, no existiendo ningún sujeto que la considerase mala, y, comparada con la de hace 1 año, el $80 \%$ dicen que es igual o mejor. Ante la pregunta acerca de cuanto le limita realizar determinadas actividades o cosas, el resultado también se muestra muy significativo siendo entre un $70 \%$ y un $98 \%$ los sujetos a los que no les limita nada realizar dichas actividades, con la excepción de los esfuerzos intensos donde a un $65 \%$ les limita un poco o mucho realizarlos. 


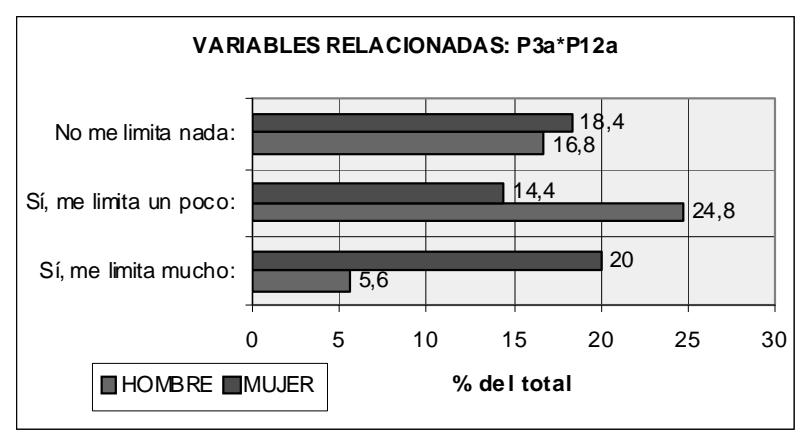

Figura 1: Resultados variables relacionadas: P3a*P12a: "Las siguientes preguntas se refieren a actividades o cosas que usted podría hacer en un día normal. Su sal ud actual, ¿ ¿Le limita hacer esas actividades o cosas?

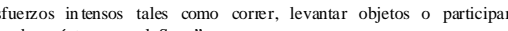
agotadores" * "Preguntas de carácter general: Sexo".

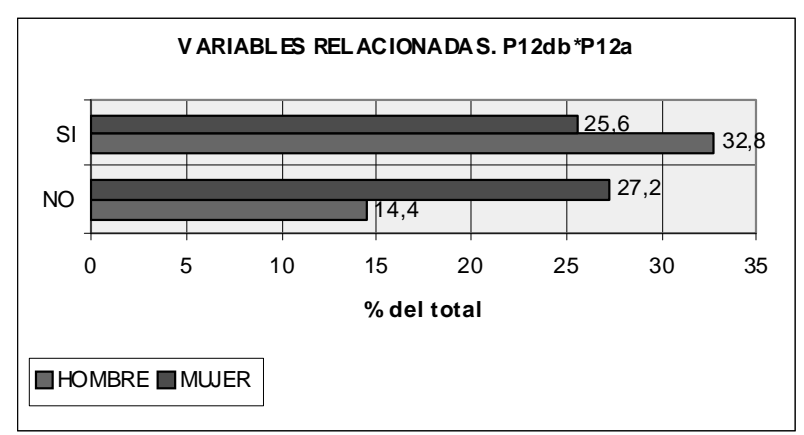

Figura 2: Resul tados variables rel acionadas: P12db*P12a: “ ¿Realiza alguna de las siguientes actividade físicas (2-3 veces por semana)?:" "Caminar rápidamente, correr, patinar, senderismo, montar en bicicleta,..." * "Preguntas de carácter general: Sexo".

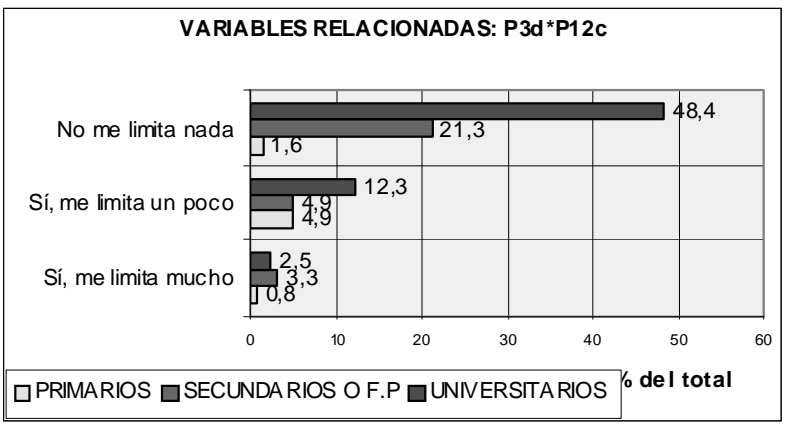

Figura 3: Resul tados variables relacionadas: P3d*P12c: "Las siguientes preguntas se refieren a actividades o es así, ¿cuánto?": "Subir varios pisos por la escalera". * “Preguntas de caráctergeneral: Nivel de estudios".

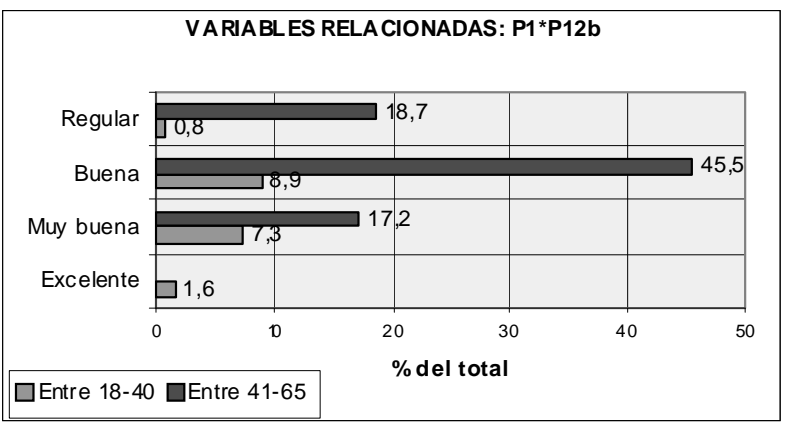

Figura 4: Resultados variables relacionadas: P1*P12b: “En general, usted diría que su salud es”: * "Preguntas de carácter general: Grupo de edad".
Entre un 70\% y un $81 \%$ de los sujetos contestaron que nunca tuvieron que reducir el tiempo dedicado a su trabajo a causa de su salud física, y entre un $63 \%$ y un $64 \%$ a causa de algún problema emocional. En cuanto a las actividades sociales a un 56\% de los sujetos, su salud física o problemas emocionales no les han dificultado nada realizarlas y a un $56 \%$ de los sujetos nunca les han dificultado sus actividades sociales.

Las preguntas relacionadas con la presencia de dolor durante la última semana un $48.8 \%$ dijeron que no tuvieron ninguno o muy poco y a un 56\% no le dificultó nada su trabajo.

Las respuestas marcadas en la pregunta acerca de cómo le han ido las cosa la última semana, indican que entre un $47 \%$ y un $48 \%$ de los trabajadores se sintieron felices, con mucha energía, calmados, tranquilos y llenos de vitalidad durante la última semana, al $51 \%$ de los sujetos les parece totalmente falso que se pongan enfermos mas fácilmente que otras personas y un $48 \%$ consideran que su salud es excelente.

Alcorrelacionar diferentes variables con la variable sexo, encontramos que al 20\% de las mujeres les limita mucho realizar esfuerzos intensos, comparado con el 5.6\% de los hombres (Figura 1) y la actividad física más realizada de manera regular por los trabajadores (caminar rápidamente, correr, patinar,....) es realizada por más hombres (41 sujetos) que por mujeres (32 sujetos) (Figura 2).

Los resultados de la correlación entre el nivel de estudios y la pregunta acerca de cuanto le limita «Subir varios pisos por la escalera», indican que a un $48 \%$ (59 sujetos) de los 77 trabajadores con estudios universitarios no le limita nada, en comparación con sólo el 1.6\% (2 sujetos) de los 9 trabajadores que tienen estudios primarios (Figura 3 ).

Otro dato a tener en cuenta hace referencia a que el $56.6 \%$ de trabajadores con estudios universitarios, solo alguna vez o nunca «Tuvieron dificultad para hacer su trabajo o sus actividades cotidianas», comparado con sólo el $1.6 \%$ de los que tienen estudios primarios. Sin embargo a la pregunta si «La salud física o los problemas emocionales le han dificultado sus actividades sociales» (como visitar a los amigos o familiares), las respuestas de los 3 grupos por niveles de estudios presentan porcentajes similares a la respuesta solo alguna vez o nunca.

Los resultados de las correlaciones por grupo de edad indican que a 29 de los 100 trabajadores con edad entre 41-65 años, y a 15 de los 23 con edad entre $18-40$ años, no les limita nada realizar «Esfuerzos intensos tales como correr, levantar objetos o participar en deportes agotadores». A la pregunta «En general, dirían que su salud es» excelente, muy buena, buena, regular o mala, el resultado mas destacado corresponde a los 24 sujetos que indicaron que es regular, siendo 23(18.7\%) de ellos de edad entre 41-65 años y sólo 1 (.8\%) de ellos de entre 18-40 años (Figura 4).

Los resultados de las correlaciones por actividad física indican que para 51 de los 73 trabajadores que realizan actividad física (2-3 veces por semana) como «Caminar rápidamente, correr, patinar, senderismo, montar en bicicleta,...m!nsu salud actual, comparada con la de hace 1 año» es mas o menos igual, igualmente un $26.4 \%$ de los trabajadores que si realizan esta actividad física afirman que no les limita nada realizar esfuerzos intensos, frente al $16.8 \%$ de los que no la realizan y que sí les limita mucho realizarla (Figura 5)

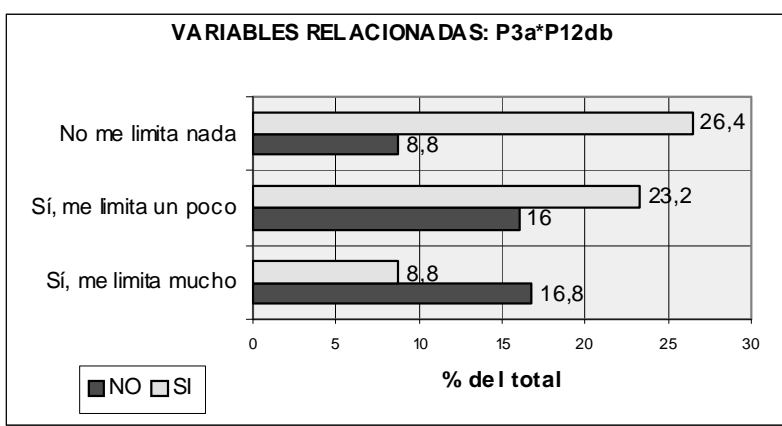

Figura 5: Resultados variables relacionadas: $\mathrm{P} 3 \mathrm{a}^{*} \mathrm{P} 12 \mathrm{db}$ : "Las siguientes preguntas se refieren a actividades o cosas que usted podría hacer en un día normal. Su salud actual, ¿Le limita hacer es as

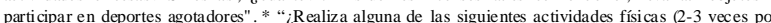
semana)?": "Caminar rápidamente, correr, pat inar, senderismo, montar en biciclet a, 
A la pregunta ¿ंTuvo dolor en alguna parte de su cuerpo durante la última semana?, donde 25 de los 73 trabajadores que realizan esta actividad física de manera regular contestaron que no tuvieron ningún dolor comparado con los 10 de 52 que no realizaban esta actividad física. Igualmente ante la pregunta ¿Hasta qué punto el dolor le ha dificultado su trabajo habitual? el 52.2\% de los trabajadores que realizan esta actividad física contestaron que un poco o nada.

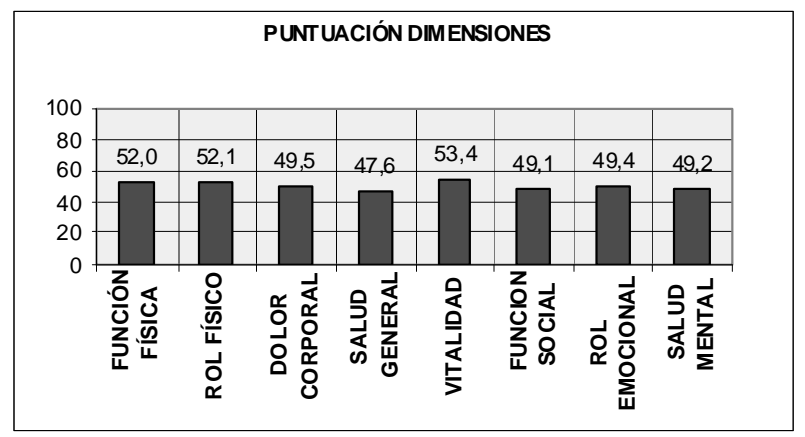

Figura 6: Resultados de la puntuación obtenida por dimensiones de los cues tionarios real izados.

Los resultados para la versión 2 del SF-36 Health Survey TM (Formularios estándar y aguda) basados en algoritmos que basan sus interpretaciones sobre la norma en base a puntuaciones (media $=50$, SD $=10$ ), indican que para todas las dimensiones el estado de salud está dentro del rango promedio, siendo las dimensiones de función física y vitalidad las que presentan una mejor puntuación (Figura 6).

En el caso de las componente sumario, ambas también se encuentran dentro del rango promedio de estado de salud, siendo la componente sumario física superior a la mental (Figura 7).

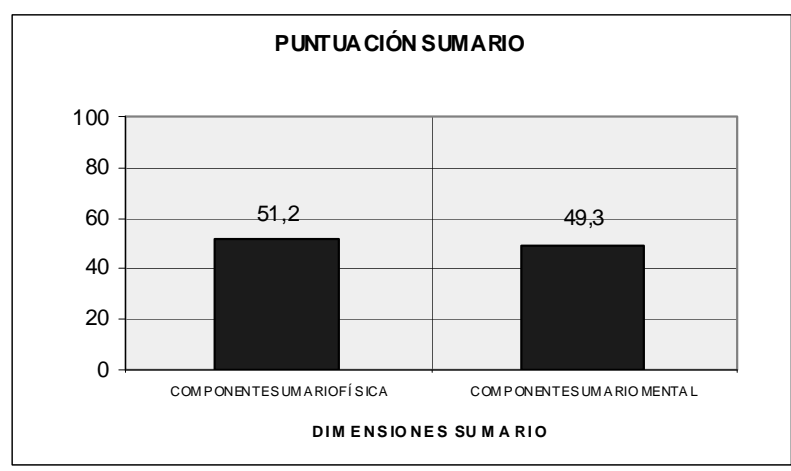

Figura 7: Resultados de la puntuación obtenida por componente sumario de los cuestionarios realizados.

\section{Discusión}

Comparando los resultados de este trabajo con los encontrados para poblaciones similares (poblaciones laborales) en la revisión bibliográfica, no podemos realizar una comparación de resultados al tratarse estos últimos de estudios comparativos con ensayos clínicos, pre y post intervención, no siendo el caso de este trabajo que ha sido realizada sobre la misma población, sin ensayo clínico y en una única intervención.

Una vez conocido que la actividad física más realizada de manera regular 2-3 veces por semana, es «Caminar rápidamente, correr, patinar, senderismo o montar en bicicleta, cabe destacar que se trata de una actividad física no reglada y sin personal cualificado que la guíe, y esto es posible que sea debido a que este tipo de actividad no está sujeto a espacio físico ni horario específico, donde la conciliación entre vida laboral y personal en los trabajadores es un factor muy tenido en cuenta.
Otro factor a tener en cuenta a la hora de elegir este tipo de actividad es la edad de los sujetos, que como hemos visto es superior a los 40 años en su mayoría, los cuales tienen una mayor conciencia de su estado de salud y de cómo mejorarla, aunque sea buscando actividades que no impliquen esfuerzos intensos.

Como se ha visto, el concepto de calidad de vida relacionado con la salud es multidimensional y está muy relacionado con los aspectos más relevantes para la satisfacción, la motivación y el rendimiento laboral de los trabajadores. La identificación de las variables y dimensiones mas relevantes para la CVRS en los trabajadores y empleados públicos, hará posible la creación de instrumentos de medida que permitan, tanto de modo global como por dimensiones, determinar los aspectos que deben ser mejorados, identificando los problemas existentes, midiendo resultados, así como realizando comparaciones para conseguir aunar los objetivos de los trabajadores con los de la empresa u organización.

Como limitaciones de este cuestionario cabría destacar queno incluye algunos conceptos de salud relacionados con la calidad de vida importantes, como trastornos del sueño, función cognitiva, aspectos familiares o la función sexual, tanto o más importantes que los ya incluidos para determinar la calidad de vida relacionada con su salud en los sujetos encuestados, ya sea bien en su vida general como laboral.

\section{Conclusión}

Dando respuesta a los objetivos planteados, en relación a la variable sexo, la única diferencia radica en que los hombres parecen tener menos limitaciones a la hora de realizar actividades que impliquen esfuerzos intensos, por grupo de edad el grupo de trabajadores de entre 18-40 años parece ser el que presenta menos limitaciones a la hora de realizar esfuerzos intensos y por nivel de estudios los trabajadores con estudios universitarios indican menos limitaciones a la hora de subir varios pisos por la escalera.

Al objetivo planteado de conocer quétipo de actividad física realizan los trabajadores del Servicio de Salud, podemos afirmar que la actividad más realizada de manera regular 2-3 veces por semana, es «Caminar rápidamente, correr, patinar, senderismo, montar en bicicleta,...».

Para conocer la relación existente entre los sujetos que realizan algún tipo de actividad física y su percepción de CVRS en su puesto de trabajo, los resultados obtenidos nos indican que los trabajadores que no realizan actividad física tienen una peor percepción de su salud o por lo menos comparada con la del año anterior, mientras que los que si realizan actividad física de manera regular presentan respuestas indicadoras de una percepción más positiva de su calidad de vida relacionada con su salud.

Las puntuaciones por dimensiones y componente sumario están en concordancia con los resultados generales mostrados de manera individual en cada ítem del cuestionario y todas las dimensiones y componentes sumarios se encuentran dentro de la media de puntuación para la población general.

Podemos concluir diciendo que a pesar de que el SF-36 es el cuestionario más utilizado en España para estudios sobre calidad de vida(Badia \& Baró, 2001), y siendo por sus propiedades psicosométricas uno de los instrumentos con mayor potencial en el campo de la CVRS (Garratt, Schmidt, Mackintosh \& Fitzpatrick, 2002). Es aplicable tanto a pacientes individuales como a la población general (Vilagut, et al., 2005), y los resultados generales nos dan atisbo de la existencia de una «deseabilidad» $\mathrm{y}$ «subjetividad» ante algunas de las respuestas.

Para futuros trabajos sería conveniente completar la evaluación de la calidadde vida relacionada con la salud, y su relación con laproductividad laboral, tanto en esta población como en otras similares a la estudiada, para así poder establecer comparaciones que nos permitan estudiar de manera mas clara el impacto de la calidad de vida en el empleado público.

\section{Referencias bibliográficas}

Alonso, J., Prieto, L., \& Antó, J. (1995). La versión española del SF-36 health survey (cuestionario de salud SF-36): Un instrumento para la medida de los resultados clínicos. Medicina Clínica (Barcelona), 
104(20), 771-776.

Amital, D., Herskovitz, C., Fostick, L., Silberman,A., Doron, Y., Zohar, J., . . \& Amital, H. (2010). The premenstrual syndrome and fibromyalgia — similarities and common features. Clinical Reviews in Allergy \& Immunology, 38(2-3), 107-107-15. doi:10.1007/s12016009-8143-0

Arévalo, M., Quintero, M.A., Sánchez, D.C., \& Palacios, L. (2005). Efectos benéficos de la modificación del estilo de vida en la presión arterial y la calidad de vida en pacientes con hipertensión. Acta Colombiana De Psicología, 2, 69-85.

Badia, X., \& Baró, E. (Atención Primaria 2001; 28: 349-356.). Cuestionarios de salud en España y su uso en atención primaria.

Boentert, M., Dziewas, R., Heidbreder, A., Happe, S., Kleffner, I., Evers, S., \& Young, P. (2010). Fatigue, reduced sleep quality and restless legs syndrome in charcot-marie-tooth disease: Aweb-based survey. Journal of Neurology, 257(4), 646-646-52. doi:10.1007/ s00415-009-5390-1

Bullinger, M., Alonso, J., Apolone, G., Leplège, A., Sullivan, M., WoodDauphinee, S., \& Bech, P. (1998). Translating health status questionnaires and evaluating their quality: The IQOLA project approach. Journal of Clinical Epidemiology, 51(11), 913-923.

Chan, W., Whitford, D., Conroy, R., Gibney, D., \& Hollywood, B. (2011). A multidisciplinary primary care team consultation in a socio-economically deprived community: An exploratory randomised controlled trial. BMC Health Services Research, 11(1), doi:10.1186/1472-6963-11-15

Gibson, J., Ivanicevich, J., Donelly, J. (1996). Las Organizaciones, (8a ed.). Madrid: McGraw Hill.

Garratt, A., Schmidt, L., Mackintosh, A., \& Fitzpatrick, R. (2002). Quality of life measurement: Bibliographic study of patient assessed health outcomemeasures. British Medical Journal,324(7351), 1417.

Graham, J., \& Streitel, K. (2010). Sleep quality and acute pain severity among young adults with and without chronic pain: The role of biobehavioral factors. Journal of Behavioral Medicine, 33:335345. doi:10.1007/s10865-010-9263-y

Hernández, R.; Fernández, C., \& Baptista, P. (2008) Metodología de la investigación ( $4^{\mathrm{a}}$ ed). México: McGraw-Hill Iberoamericana.

Huisinga, J., Filipi, M., \& Stergiou, N. (2011). Elliptical exercise improves fatigue ratings and quality of life in patients with multiple sclerosis. Journal of Rehabilitation Research and Development, 48(7), 881881-889.

Iraurgi Castillo, I. (2007). Comparación del instrumento de salud SF-12 frente al SF-36 en pacientes en mantenimiento con metadona. Adicciones: Revista De Socidrogalcohol, 19(1), 59-67.

Koller, H., Reynolds, J., Zenner, J., Forstner, R., Hempfing, A., Maislinger, I., . . \& \& Hitzl, W. (2009). Mid- to long-term outcome of instrumented anterior cervical fusion for subaxial injuries. European Spine Journal, 18(5), 630-630-53. doi:10.1007/s00586008-0879-3

Láinez, M., Domínguez, M., Rejas, J., Arriaza, E., García-García, M., \& Palacios, G. (2007). Impacto de distintas enfermedades en la calidad de vida relacionada con la salud (CVRS) en una población laboral. Anales de Medicina Interna, 24, 3-11.

Leme, J., \& Meyer, E. (2008). Efectos de la gimnasia laboral en la calidad de vida de los trabajadores; Effects of laboral gymnastics on workers health. Ciencia \& Trabajo, 10(29), 100-105.

León Prados, J.A., Fuentes García, I., González Jurado, J.A., Fernández, A., Costa, E., \& Ramos, A. (2011). Actividad física y salud percibida en un sector de la población sevillana: Estudio piloto. Revista Internacional De Medicina y Ciencias De LaActividad Física y Del Deporte, 41, 10-16.

Liu, W., Wu, X., Guo, J., Zhuang, S., \& Teng, G (2010). Long-term outcomes of patients with lumbar disc herniation treated with percutaneous discectomy: Comparative study with microendoscopic discectomy. Cardiovascular and Interventional Radiology, 33(4), 780-780-6. doi:10.1007/s00270-009-9720-6
Mariconda, M., Galasso, O., Attingenti, P., Federico, G., \& Milano, C. (2010). Frequency and clinical meaning of long-term degenerative changes after lumbar discectomy visualized on imaging tests. European Spine Journal, 19(1), 136-136-43. doi:10.1007/s00586009-1201-8

Martínez, F.S., Cocca, A., Mohamed, K., \& Ramírez, J.V. (2010). Actividad física y sedentarismo: Repercusiones sobre la salud y calidad de vida de las personas mayores. Retos. Nuevas Tendencias EnEducación Física, Deporte y Recreación, 17, 126-129.

Rodríguez Vidal, M., Merino Escobar, M., \& Castro Salas, M. (2009). Psychometric assesment of the physical and mental components of the sf-36 in chronic renal insufficiency failure patients in treatment with haemodyalisis. Ciencia y Enfermería, 15(1), 75-88.

Rodrýguez-Marýn, J. ( 2010.) Quality of working life of health professionals. Calidad de vida laboral en profesionales de la salud. Revista de Calidad Asistencial. doi: 10.1016/j.cali.2010.09.002.

Santos, A.C., Bredemeier, M., Rosa, K.F., Amantea, V.A., \& Xavier, R.M.(2011). Impact on the quality of life of an educational program for the prevention of work-related musculoskeletal disorders: A randomized controlled trial. BMC Public Health, 11, 60-60. doi:10.1186/1471-2458-11-60

Schultz, I.Z., Crook, J., Berkowitz, J., Milner, R., Meloche, G.R., \& Lewis, M.L. (2008). A prospective study of the effectiveness of early intervention with high-risk back-injured workers-A pilot study. Journal of Occupational Rehabilitation, 18(2), 140-51. doi: 10.1007/s10926-008-9130-7

Segura, M., \& Espejo, J. (2008). Salud percibida por los pacientes en DPCA y DPA. Revista Sociedad española de enfermería y nefrología, 11(2): 102-109.

Urzúa M,A., \& Caqueo-Urízar,A. (2012). Calidad de vida: Una revisión teórica del concepto. Terapia Psicológica, 30(1), 61-71.

Vilagut, G., Ferrer, M., Rajmil, L., Rebollo, P., Permanyer-Miralda, G., Quintana, J.M., \& Alonso, J. (2005). The spanish version of the short form 36 health survey: A decade of experience and new developments. Gaceta Sanitaria, 19(2), 135-150.

Vilagut, G., Valderas, J., Ferrer, M., Garin, O., López-García, E., \& Alonso, J. (2008). [Interpretation of SF-36 and SF-12 questionnaires in spain: Physical and mental components]. Medicina Clínica, 130(19), 726-735.

Ware Jr, J.E. (2000). SF-36 health survey update. Spine, 25(24), 3130.

WHOQOL-OMS, (1996). Evaluación de la calidad de vida. ¿Por qué calidad de vida?; 1994. Foro Mundial De La Salud, OMS, Ginebra, 28.

Wolinsky, F., Malmstrom, T., Miller, J., Andresen, E., Schootman, M., \& Miller, D. (2009). Antecedents of global decline in health-related quality of life among middle-aged african americans. The Journals of Gerontology, 64B(2), 290-290-5.

Zanini, C., Jardim, P., Salgado, C., Nunes, M., Urzêda, F., Carvalho, M., ... \& Souza, W. (2009). O efeito da musicoterapia na qualidade de vida e na pressão arterial do paciente hipertenso; music therapy effects on the quality of life and the blood pressure of hypertensive patients; el efecto de la musicoterapia en la calidad de vida y en la presión arterial del paciente hipertenso. Arquivos Brasileiros de Cardiologia, 93(5), 495-540.

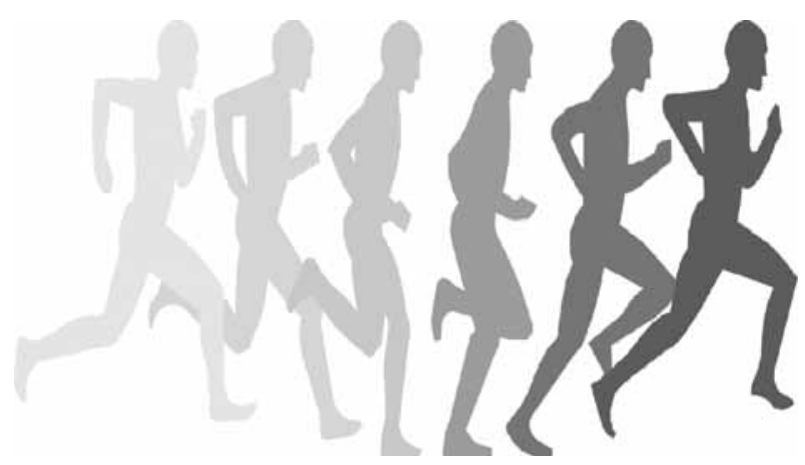

